\title{
Self-Perceived Confidence of Medical Students Communicating with Pediatric Patients in a 7-Week Pediatric Placement: A Pilot Survey
}

This article was published in the following Dove Press journal:

Advances in Medical Education and Practice

\author{
Jhia Jiat Teh $\mathbb{D}^{\prime}$ \\ Ka Yan Cheung (D) \\ Yusuf Patrick' \\ Mona Panahi id \\ Robert Boyle 2 \\ Gareth Tudor-Williams ${ }^{2}$ \\ 'Department of Medicine, Imperial \\ College London, London, UK; \\ ${ }^{2}$ Department of Pediatrics, Imperial \\ College London, London, UK
}

Correspondence: Jhia Jiat Teh

School of Medicine, Imperial College

LondonExhibition Road, London SW7

2AZ, UK

Tel +44 $742132 \quad 1772$

Email jhia.teh I5@imperial.ac.uk
Background: Pediatrics is a specialty reserved until later stages of the medical curriculum, with many students receiving early exposure via volunteering opportunities. Self-perceived confidence across the pediatric curriculum is crucial, due to limited clinical exposure before qualification. We aimed to assess the impact of a 7-week pediatric placement on medical students' self-perceived confidence and factors that influenced self-perceived confidence.

Methods: We conducted a prospective pilot survey on three cohorts of fifth-year students undertaking pediatric placements in 2018. A two-part questionnaire was distributed before and after the placement, evaluating the level of self-confidence in clinical skills using a 10-point scale. Results: Of 103 students, $62(60 \%)$ students completed both questionnaires. Of these, 34 $(55 \%)$ students reported previous professional experiences with children. There was a significant increase in self-reported confidence scores across ten questions before (mean 5.4 [IQR 4.1-6.1]) and after the placement (7.6 [6.6-8.5], $p<0.0001)$. Subgroup analyses between students with prior professional experience with children and those without revealed a significant difference in preplacement confidence in four pediatric practices: verbal communication, physical engagement, asking sensitive or probing questions, and explaining medical management $(p<0.05)$. There was no significant difference in postplacement confidence between these two groups.

Conclusion: Medical students with prior professional experience with children reported higher self-confidence in interacting with pediatric patients prior to placement. However, a large and consistent increase in confidence across the cohort was such that there were no measurable differences at exit. This study supports the value of undergraduate pediatric training in promoting student self-confidence in managing pediatric clinical issues.

Keywords: medical education, pediatrics, self-confidence, medical students

\section{Introduction}

Pediatrics is a specialty often reserved for the later years of the medical curriculum, with clinical experience being limited to one placement stretching over several weeks. Despite this, many medical students receive exposure to pediatrics in both nonclinical and clinical settings prior to starting placements through voluntary or (more rarely) paid work. These individuals are often perceived by their supervisors to have a higher level of confidence and competence upon starting their attachment. However, no formal research has been undertaken into this matter.

Low self-confidence has been linked to higher levels of anxiety and increased burnout, which are increasingly common in the modern National Health Service 
(NHS) in the UK. ${ }^{1,2}$ Poor self-confidence in communication may render children and carers unable to understand their options, and more importantly this may influence physicians' ability to engage in shared decision-making. Although self-reported confidence has not been formally associated with competence in practice, uncertainty about how to manage pediatric presentations is common in junior doctors, and confidence-building may be important to incorporate in undergraduate medical school pediatric curricula.

The confidence levels of medical students upon entering and leaving their pediatric rotation are important, as it is the only formal placement many will attend before potentially working in this specialty during foundation training and beyond. Students at Imperial College London undertake a predetermined 7-week pediatric rotation in urban hospitals as part of the fifth-year curriculum in the penultimate year of medical studies. The pediatric placement consists of regular emergency department and inpatient and outpatient medical and surgical interactions with attending physicians and residents. Summative examinations take place in summer at the end of the academic year, where students are assessed using a combination of written examinations and Practical Assessment of Clinical Examination Skills (PACES).

The role of self-perceived confidence among medical students is vital to explore, since the concept of confidence is closely linked with Bandura's concept of self-efficacy. ${ }^{3}$ Therefore, an effective pediatric placement should lead to similar levels of self-confidence, regardless ofexperience. This influences learners' effectiveness and possibly measured performance in PACES pediatric stations. If experience were a key component to the level of self-confidence before and more importantly after a fifth-year pediatric placement, this would open an avenue for developing programs to facilitate professional contact with children prior to fifth-year placements.

The primary aim of this study was to assess medical students' self-perceived confidence in regard to the communication domain of pediatric practice both before and after undertaking a 7-week pediatric placement across different hospital sites at Imperial College London. To our knowledge, this is the first pilot study investigating self-reported confidence in communication skills in pediatric practice during a pediatric placement in one medical school. A secondary outcome of this study was to assess the extent of modifiable and unmodifiable factors that affect self-confidence toward pediatric patients, both prior to and after the placement.

\section{Methods}

\section{Study Design}

A pilot cohort study was conducted between July 23 and December 15, 2018. Participants were self-selected from year 5 students scheduled to undertake their 7-week pediatric placement during this period, when it would not interfere with revision for the examinations. The pediatric placement consisted of regular rotations between the pediatric emergency department and inpatient and outpatient urban settings under a team of attending physicians and residents. There were no exclusion criteria for participants, with students being recruited via posttutorial announcements at the beginning of their rotation.

\section{Study Protocol}

This study was reviewed by the Medical Education Ethics Committee (MEEC) at Imperial College London. Ethical approval was obtained (MEEC reference 171866). Participants were asked to complete two questionnaires. The first was completed after the introductory session for the pediatric rotation, and sought information on personal particulars (age, sex, marital status, any siblings, any children, extended family below the age of 10 years, participation in extracurricular activities involving children; Suppl Figure 1). In addition, participants were asked to rank their selfperceived confidence in ten clinical areas (communicating with children, physically engaging with children, asking children sensitive or probing questions, performing basic medical procedures, interacting with parents, explaining medical conditions to children, explaining medical management to children, explaining medical conditions to parents, explaining medical management to parents, recognizing a sick child; Suppl Figure 2). The questionnaire was constructed to determine levels of confidence in interacting with pediatric patients and their carers as implied in the national child health curriculum for medial undergraduates outlined by the Royal College of Pediatrics and Child Health. ${ }^{4}$ The questions were reviewed and refined by an iterative process involving consultations with final-year medical students and the faculty.

The second questionnaire was a repeat of self-perceived confidence in ten clinical areas, and was carried out in the final week of the 7-week placement. Both questionnaires included a question pertaining to participants' email 
addresses so their answers could be paired, and data were anonymized prior to analysis. All participants provided consent before starting the first questionnaire and were free to exit the study at any time without having to give a reason. Self-perceived confidence scales were not used as a surrogate for formal competence-based assessment, as it has previously been indicated that self-confidence and academic performance are not linked. ${ }^{5-8}$ Instead, selfconfidence was determined to be an important attribute and outcome objective on undergraduate pediatric courses by allowing students to access the full range of training opportunities and to fully engage in their work with children as junior doctors.

\section{Statistical Methods}

Descriptive statistics were used for all responses. Normality for self-confidence scores was evaluated with the D'Agostino-Pearson test. Nonparametric statistical analyses were performed using GraphPad Prism 8.0 (GraphPad Software, San Diego, CA), since selfconfidence scores were not normally distributed. Paired self-confidence data pre- and postplacement were compared using Wilcoxon's signed-rank test. To determine whether modifiable and unmodifiable features were associated with self-reported confidence scores across all ten questions, the Mann-Whitney $U$ test and Kruskal-Wallis test were used. All $p$-values are two-tailed, and $p<0.05$ was considered significant.

\section{Results}

\section{Participant Characteristics}

Figure 1 summarizes the recruitment process for study participants. The characteristics of 62 participants who completed both questionnaires are summarized in Table 1. Participants' mean age was $22.7 \pm 1.9$ years, and $38(61 \%)$ were male. None of the participants was married or had any children. A total of $53(85 \%)$ had at least one sibling, and $39(63 \%)$ reported having at least one extended family member $<10$ years old. Nine (15\%) students reported participation in the early-life patient-contact program.

One or more experiences with children in professional settings was reported by 34 students (55\%), and a detailed quantitative analysis was performed (Table 2). The median cumulative duration of experience with children in professional settings was 122 hours. Based on the open-ended response, these experiences were categorized into four
Medical students undertaking the first three pediatric placements $(n=148)$

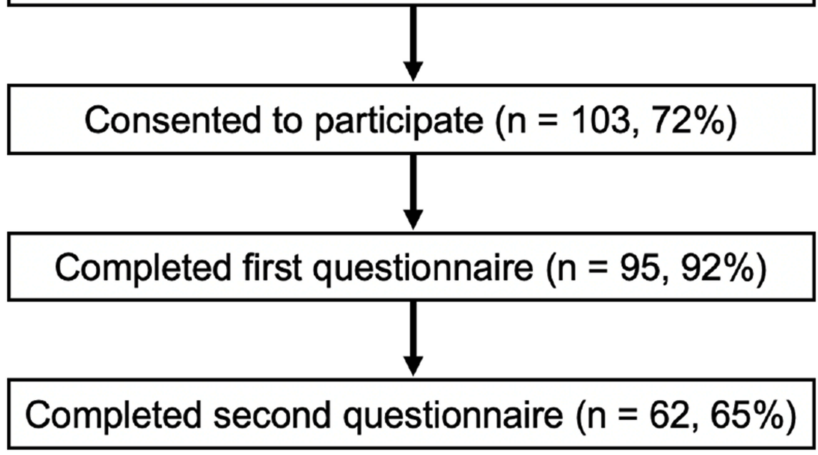

Figure I Flowchart illustrating recruitment process for study participants. Percentages indicate the proportion of individuals who remained from the previous stage.

themes. Over half the responses were categorized as "teaching and tutoring", followed by "pediatric ward experience" (38\%), "leadership and care" (29\%), and "medical experience in the community" $(12 \%)$.

\section{Self-Reported Confidence}

We compared self-reported confidence across ten questions addressing different aspects of pediatric competence before and after pediatric placement (Table 3, Suppl Figure 3). Participants improved their confidence levels across all questions on the questionnaire (mean difference $2.4,95 \%$ CI 2.0-2.7; $\mathrm{p}<0.0001)$. Of the ten questions, we observed the largest improvement in confidence in "recognizing a sick child", with a mean difference of 3.5. Before pediatric placement, two questions assessing confidence in "demonstrating basic medical procedures" and "explaining medical

Table I Demographics of Study Participants $(n=62)$

\begin{tabular}{|l|l|}
\hline & $\mathbf{n}(\%)$ \\
\hline Age, mean (SD), years & $22.7(1.9)$ \\
Married & $38(6 \mathrm{I})$ \\
& 0 \\
\hline Number of siblings & \\
0 & $9(15)$ \\
$\mathrm{I}$ & $28(45)$ \\
2 & $14(23)$ \\
$\geq 3$ & $\mathrm{II}(18)$ \\
\hline Extended-family members <10 years & $39(63)$ \\
Previous experience with children in professional & $34(55)$ \\
settings & \\
Participation in early-life patient-contact program & $9(15)$ \\
\hline
\end{tabular}

Note: Data are number (percentage) of individuals unless otherwise indicated. 
Table 2 Detailed Analyses of Previous Experience with Children in Professional Settings $(n=34)$

\begin{tabular}{|l|l|}
\hline & n (\%) \\
\hline $\begin{array}{l}\text { Male sex } \\
\text { Cumulative time, median (IQR), hours }\end{array}$ & $18(53)$ \\
\hline $\begin{array}{l}\text { Cumulative time, hours } \\
<100\end{array}$ & $16(48-240)$ \\
$100-500$ & $11(32)$ \\
$>500$ & $7(21)$ \\
\hline $\begin{array}{l}\text { Nature of the experience* (n=45) } \\
\text { Teaching and tutoring }\end{array}$ & $18(53)$ \\
Leadership and care & $10(29)$ \\
Medical experience in the community & $4(12)$ \\
Pediatric ward experience & $13(38)$ \\
\hline
\end{tabular}

Notes: *Categorized into four groups:

(i) Teaching and tutoring: any paid teaching work with children

(ii) Leadership and care: any roles, including summer camps (eg, Rainbows and Brownies), youth leaders at church, and nannies

(iii) Medical experience in the community: any clinical experience outside the hospital, such as first-aid teaching and working with children with learning difficulties

(iv) Pediatric ward experience: such schemes as Imperial College Pediatrics Society PlayTeam or any previous medical pediatric ward experience.

management" to children received the lowest scores (4.3 \pm 2.2 and $4.3 \pm 2.0$ respectively). After placement, the domain assessing self-confidence in "explaining medical management" to children received the lowest score $(6.7 \pm 1.8)$.

Subgroup analyses focused on differences in selfreported confidence with respect to modifiable and unmodifiable factors, such as age, sex, number of siblings, and young extended-family members. Statistical analyses demonstrated no significant difference in confidence scores before pediatric placement with respect to each of the unmodifiable factors. Subsequent analyses focused on modifiable factors, such as participants who had had experience working with children in professional settings compared to those who had not. Overall, there was no significant difference in end-of-placement self-reported confidence between the two groups, and although confidence scores preplacement were higher in those with professional experience with children, this difference was not statistically significant (mean difference $0.66,95 \% \mathrm{CI}-1.32$ to 0.10 ).

The first hypothesis, ie, that before pediatric placement there would be a significant difference in self-perceived confidence toward pediatric patients between students who had had no experience working with children compared to those who had had experience working with children in professional settings, was analyzed using Mann-Whitney $U$ test. Students who had had professional experience with children reported higher self-confidence scores on four of ten questions. These included confidence in verbally communicating with children, physically engaging with children, asking sensitive or probing questions of children, and explaining medical management to children $(p<0.05$, Table 4$)$.

The second hypothesis, ie, that after pediatric placement there would be no significant difference in selfperceived confidence toward pediatrics patients between students who had had no experience working with children in a professional setting compared to those who had not, was tested using Mann-Whitney $U$ test. There were no significant differences in confidence levels between these two groups of participants.

\section{Discussion}

It is widely acknowledged that communicating with children and their families can pose a challenge for health-care professionals. In pediatrics, this medical relationship and communication involves three members - physician,

Table 3 Self-Reported Confidence Scores Before and After Placement $(n=62)$

\begin{tabular}{|l|l|l|l|}
\hline & Preplacement & Postplacement & Mean Difference (95\% CI) \\
\hline I. I am confident in verbally communicating with children. & $5.9(2.5)$ & $8.2(1.3)$ & $2.1(1.6-2.7)$ \\
2. I am confident physically engaging with children. & $5.7(2.4)$ & $7.9(1.3)$ & $2.1(1.5-2.7)$ \\
3. I am confident with asking sensitive or probing questions of children. & $4.5(2.3)$ & $6.8(2.0)$ & $2.2(1.7-2.8)$ \\
4. I am confident demonstrating basic medical procedures to children. & $4.3(2.2)$ & $7.1(1.8)$ & $2.8(2.3-3.3)$ \\
5. I am confident interacting with parents. & $5.6(2.1)$ & $8.5(1.0)$ & $2.8(2.3-3.4)$ \\
6. I am confident explaining medical conditions to children. & $4.8(2.1)$ & $6.8(1.7)$ & $2.1(1.6-2.5)$ \\
7. I am confident explaining medical management to children. & $4.3(2.0)$ & $6.7(1.8)$ & $2.4(1.9-2.9)$ \\
8. I am confident explaining medical conditions to parents. & $5.9(I .9)$ & $7.8(1.3)$ & $1.9(I .4-2.4)$ \\
9. I am confident explaining medical management to parents. & $5.8(I .9)$ & $7.7(I .4)$ & $1.9(I .4-2.4)$ \\
I0. I am confident that I can recognize a sick child. & $4.4(I .7)$ & $7.9(1.2)$ & $3.6(3.1-4.0)$ \\
\hline
\end{tabular}

Notes: Values expressed as means (SD). All self-reported confidence scores postplacement were significantly higher than scores from preplacement using Wilcoxon signedrank test $(p<0.0001)$. 
Table 4 Comparison of Self-Reported Confidence Scores Before Placement in Participants with or Without Professional Experience with Children

\begin{tabular}{|l|l|l|l|}
\hline & Yes & No & p-value* \\
\hline I. I am confident in verbally communicating with children. & $6.6(2.4)$ & $5.4(2.1)$ & 0.018 \\
2. I am confident physically engaging with children. & $6.4(2.3)$ & $5.0(2.1)$ & 0.017 \\
3. I am confident with asking sensitive or probing questions of children. & $5.0(2.1)$ & $4.0(2.3)$ & 0.049 \\
4. I am confident demonstrating basic medical procedures to children. & $4.3(2.1)$ & $4.2(2.4)$ & 0.825 \\
5. I am confident interacting with parents. & $5.5(2.2)$ & $5.7(2.0)$ & 0.792 \\
6. I am confident explaining medical conditions to children. & $5.1(2.1)$ & $4.4(2.2)$ & 0.130 \\
7. I am confident explaining medical management to children. & $4.7(2.0)$ & $3.9(1.9)$ & 0.04 I \\
8. I am confident explaining medical conditions to parents. & $6.0(2.0)$ & $5.7(1.9)$ & 0.620 \\
9. I am confident explaining medical management to parents. & $6.0(1.9)$ & $5.5(2.1)$ & 0.420 \\
I0. I am confident that I can recognize a sick child. & $4.3(1.9)$ & $4.5(I .6)$ & 0.650 \\
Mean & $5.4(I .5)$ & $4.8(I .5)$ & 0.095 \\
\hline
\end{tabular}

Notes: Values expressed as means (SD). *Two-tail Mann-Whitney $U$ test was used.

family, and child — leading to an increasing degree of communication difficulty. ${ }^{9,10}$ A survey by the UK Healthcare Commission reported that many children aged 0-17 were dissatisfied with the way their health-care professionals related to them while they were admitted. ${ }^{11}$ Communicating with a child is complex and often influenced by the developmental and cognitive stage of the child. ${ }^{9}$ Effective communication can not only enhance patient outcomes and satisfaction but also yield greater professional satisfaction in clinical practice. ${ }^{12,13}$ On the other hand, low self-confidence reported in communicating with pediatric patients may positively reinforce learners to become ineffective communicators. ${ }^{14}$ This may be reflected in future consultations with distressed parents and their children as practicing junior residents. Communication is also a key driver of patient-safety events, and a lack of effective communication may create situations where medical error can occur, which can negatively impact patient outcomes in the long term. ${ }^{15}$

In the majority of medical institutions in UK, pediatric attachment is often reserved for later years of the clinical curriculum. For medical students, the attachment is likely to be their first and last contact with pediatric patients and their carers until they qualify as a practising clinician. Conventionally, those with pediatric experience are often perceived by senior pediatricians to demonstrate a higher level of confidence in communicating with pediatric patients and their carers. However, the level of perceived confidence in clinical communication between students, patients, and their families has not been formally assessed.

One of the aims of the study was to compare students' self-reported confidence in communication within pediatric practice as they progressed through the placement. We observed a significant increase in self-reported confidence levels across all nine questions in the communication domain in pediatric practice following the 7 -week placement. The same trend was observed assessing the participants' confidence in being able to recognize a sick child. We anticipated an increase in self-confidence levels regardless of experience, since this demonstrates effective pediatric placement that empowers medical students to be involved in pediatric care. Self-confidence has been defined as the belief in one's ability to accomplish a goal or task. ${ }^{16}$ While it does not necessarily correlate with competence, it is nevertheless an important prerequisite for students to be able to fully engage in clinical activities during medical placement. $^{8}$

In our study, we found that before pediatric placement, students who had had experience working with children in professional settings reported significantly higher confidence levels in four of ten questions: verbally communicating with children, physically engaging with children, asking sensitive or probing questions of children, and explaining medical management to children. This can potentially be explained by the assumption that those who have had experience with children will be better equipped with the verbal and nonverbal skills to engage interactively with them. A significant number of skills are required in approaching and communicating with younger children, taking into account the patient's developmental stage. ${ }^{17,18}$ Furthermore, it is interesting to note that between these two groups, there was no significant difference in self-reported confidence scores on any of the ten questions after pediatric placement. This indicates that the medical curriculum and 
course structure have successfully brought students to a standardized level of self-perceived confidence, regardless of experience.

Over half the cohort reported prior contact with children in professional settings. The majority of the professional experience with children was of an educational nature, comprised of tutoring and teaching, while experience in the pediatric ward constituted more than a third of participants' experience. At Imperial College London, students may participate in volunteering organizations outside academic work. In particular, "PlayTeam" and "Teddy Bear Hospital" are two popular volunteering programs that facilitate regular contact with children in either pediatric wards or local primary schools. A small cohort of students were offered the opportunity by the college to participate in the voluntary, unassessed early-life patient-contact program, which involves regularly observing child development from prebirth to the early years longitudinally. ${ }^{19}$ The potential benefits of these programs in promoting interest in pediatrics as a specialty and self-confidence in interacting with children in the clinical setting remain to be assessed in the future by means of longitudinal studies.

One important aspect of this study is the assessment of self-confidence instead of actual demonstrated competence or performance within various domains of pediatric practice. The questionnaire addressed the areas of communicating and physically engaging with children, interacting with parents, explaining medical conditions and management to both parties, and recognizing a sick child. Within the literature, there are discrepancies in the correlation between self-reported confidence and clinical competence in performing medical tasks. A lack of agreement was found between pretest confidence and subsequent task performance in three common clinical pediatric skills. ${ }^{6}$ Among junior doctors, Barnsley et al reported no relationship between self-reported level of confidence and observed competence for a range of routine, procedural clinical skills. ${ }^{8}$ It is important to note that these studies assessed the correlation between self-reported confidence and competence in clinical skills. The relationship between confidence, competence, and performance has not been explored in our study. Although self-reported confidence may not indicate actual competence or performance, students who report a lack of self-assurance are presumably more likely to perform subpar compared to their confident counterparts, and thus may find meeting the needs of patients more challenging. Negative self-appraisal and feelings of incompetence belong to reduced personal accomplishment, which is one factor in the three-dimensional construct conceptualized by workplace burnout. $^{20,21}$ In addition, such factors as personality traits and personal circumstances may determine the extent to which chronic stress leads to burnout. Since physician burnout is an increasing phenomenon seen in health-care professionals, greater emphasis should be placed on tackling students' perceived clinical accomplishment before they enter the workplace.

Although this study offers insight into the level of selfconfidence in interacting with pediatric patients and carers among undergraduate medical students, we would like to point out a number of limitations. Firstly, since this was a pilot single-institution study, there were no sample-size calculations. The reproducibility of the results remains to be addressed in other medical institutions. Secondly, since the characteristics of nonparticipants were not captured, potential differences in reported self-confidence in elements of the pediatric placement in the nonparticipant cohort may have existed. This may be attributed to such reasons as lack of participatory interest due to an inability to recognize the relevance of the study or uncertainty toward reflecting own's confidence before the placement. In addition, the self-perceived confidence of a small percentage of attrited participants in the postplacement questionnaire was not measured. This may have exaggerated the positive change in self-confidence scores after placement. Finally, there are many influences on self-reported confidence toward interaction in pediatric practice. Therefore, it is difficult to capture other possible unidentified confounding factors that may positively or negatively affect participants' self-reported confidence. While clinical competence in pediatrics is formally assessed via summative examinations, such as PACES and written assessments, self-confidence in communicating with pediatric patients could also be incorporated as a formal teaching objective in the pediatric curriculum.

\section{Conclusion}

In this first survey of self-reported confidence among undergraduate medical students training in pediatric placement, we found differences at baseline related to pediatric experience, but a large and consistent increase in confidence across all groups such that there were no differences at exit based on experience with children. This study supports the value of undergraduate pediatric training in promoting student self-confidence in managing pediatric clinical issues. Further investigations could identify if a relationship exists between a student's self-perceived confidence and actual competence in pediatric practice assessed by summative written or practical exams. 


\section{Acknowledgments}

The authors would like to thank all the medical students who participated in this study.

\section{Ethics Approval and Consent to Participate}

Information regarding the study was provided to all participants, and participation in the questionnaire study was voluntary. Ethics approval was obtained from the Medical Education Ethics Committee (MEEC) at Imperial College (MEEC 1718-66).

\section{Disclosure}

The authors declare that the manuscript was recently presented as a poster presentation at Developing Excellence in Medical Education Conference (DEMEC) 2019. There are no other conflicts of interest. The authors alone are responsible for the content and writing of this article.

\section{References}

1. Yu JH, Chae SJ, Chang KH. The relationship among self-efficacy, perfectionism and academic burnout in medical school students. Korean J Med Educ. 2016;28(1):49-55. doi:10.3946/kjme.2016.9

2. Villacieros M, Olmos R, Bermejo JC. The empathic process and misconceptions that lead to burnout in healthcare professionals. Span J Psychol. 2017;20:E68. doi:10.1017/sjp.2017.66

3. Bandura A. Self-efficacy mechanism in human agency. Am Psychol. 1982;37(2):122-147. doi:10.1037//0003-066X.37.2.122

4. Royal College of Paediatrics and Child Health. Undergraduate Curriculum for Child Health - November 2015; 2015.

5. Morgan PJ, Cleave-Hogg D. Comparison between medical students' experience, confidence and competence. Med Educ. 2002;36 (6):534-539. doi:10.1046/j.1365-2923.2002.01228.x

6. O’Donoghue D, Davison G, Hanna L, McNaughten B, Stevenson M, Thompson A. Calibration of confidence and assessed clinical skills competence in undergraduate paediatric OSCE scenarios: a mixed methods study. BMC Med Educ. 2018;18(1):211. doi:10.1186/s12909-018-1318-8
7. Liaw SY, Scherpbier A, Rethans J, Klainin-Yobas P. Assessment for simulation learning outcomes: a comparison of knowledge and self-reported confidence with observed clinical performance. Nurse Educ Today. 2012;32(6):35. doi:10.1016/j.nedt.2011.10.006

8. Barnsley L, Lyon PM, Ralston SJ, et al. Clinical skills in junior medical officers: a comparison of self-reported confidence and observed competence. Med Educ. 2004;38(4):358-367. doi:10.1046/ j.1365-2923.2004.01773.x

9. Mărginean CO, Meliţ LE, Chinceşan M, et al. Communication skills in pediatrics - the relationship between pediatrician and child. Medicine. 2017;96(43):e8399. doi:10.1097/MD.0000000000008399

10. Crossley J, Eiser C, Davies HA. Children and their parents assessing the doctor-patient interaction: a rating system for doctors' communication skills. Med Educ. 2005;39(8):820-828. doi:10.1111/j.13652929.2005.02230.x

11. Ramm J, Reeves R, Graham C Patient survey report 2004 - young patients. 2004.

12. Lewis CC, Pantell RH, Sharp L. Increasing patient knowledge, satisfaction, and involvement: randomized trial of a communication intervention. Pediatrics. 1991;88(2):351-358.

13. Levetown M. Communicating with children and families: from everyday interactions to skill in conveying distressing information. Pediatrics. 2008;121(5):1441. doi:10.1542/peds.2008-0565

14. Hecimovich MD, Volet SE. Importance of building confidence in patient communication and clinical skills among chiropractic students. J Chiropr Educ. 2009;23(2):151-164. doi:10.7899/1042-50 55-23.2.151

15. O'Daniel M, Rosenstein AH. Professional communication and team collaboration. In: Hughes RG, editor. Patient Safety and Quality: An Evidence-Based Handbook for Nurses. Rockville (MD): Agency for Healthcare Research and Quality (US); 2008:271-281.

16. Potter PA, Perry AG, Hall AM, Stockert PA (editors), Ochs G. Fundamentals of nursing. 9th ed. Elsevier: 2017.

17. Christie D, Viner R. Adolescent Development. London; 2005.

18. Bell J, Condren M. Communication strategies for empowering and protecting children. J Pediatric Pharmacol Therapeutics. 2016;21 (2):176-184. doi:10.5863/1551-6776-21.2.176

19. Imperial College London. Follow my footsteps. Available from: https://www.imperial.ac.uk/medicine/study/undergraduate/follow-my -footsteps/. Accessed June 29, 2019.

20. Maslach C, Jackson SE, Leiter MP. Maslach Burnout Inventory Manual. Palo Alto, CA: Consulting psychologists press; 1996.

21. Patel RS, Bachu R, Adikey A, Malik M, Shah M. Factors related to physician burnout and its consequences: a review. Behav Sci. 2018;8 (11). doi:10.3390/bs8110098

\section{Publish your work in this journal}

Advances in Medical Education and Practice is an international, peerreviewed, open access journal that aims to present and publish research on Medical Education covering medical, dental, nursing and allied health care professional education. The journal covers undergraduate education, postgraduate training and continuing medical education including emerging trends and innovative models linking education, research, and health care services. The manuscript management system is completely online and includes a very quick and fair peer-review system. Visit http://www.dovepress.com/testimonials.php to read real quotes from published authors. 